University of Wollongong

Research Online

Australian Institute for Innovative Materials -

Papers

Australian Institute for Innovative Materials

$1-1-2018$

Electrochemically in situ controllable assembly of hierarchically-ordered and integrated inorganic-carbon hybrids for efficient hydrogen evolution

Zhongli Wang

National Institute for Materials Science

Keju Sun

Yanshan University

Joel Henzie

National Institute for Materials Science

Xianfeng Hao

Yanshan University

Yusuke Ide

National Institute for Materials Science

See next page for additional authors

Follow this and additional works at: https://ro.uow.edu.au/aiimpapers

Part of the Engineering Commons, and the Physical Sciences and Mathematics Commons

Research Online is the open access institutional repository for the University of Wollongong. For further information contact the UOW Library: research-pubs@uow.edu.au 


\title{
Electrochemically in situ controllable assembly of hierarchically-ordered and integrated inorganic-carbon hybrids for efficient hydrogen evolution
}

\begin{abstract}
Inorganic-carbon hybrid materials are an emerging class of nanostructured catalysts that can enhance various energy-oriented electrochemical reactions. Despite recent progress, it is still very challenging to controllably generate the hybrid carbon architecture and its inorganic components with a single approach. Inspired by the flexible redox properties of conductive polyaniline (PANI) polymer, we develop a redox-unit cooperative assembly strategy to synthesize hierarchically-ordered and integrated inorganic-carbon hybrids by electrochemically constructing the nanostructures of PANI and then modifying their redox states to controllably bond different metal complexes. The needle-branched PANI nanofibers are assembled in situ into a three-dimensional (3D) hierarchical framework on carbon paper by an anion induced electrochemical polymerization. Interestingly, tuning the redox states of PANI with a potentiostatic method achieves a controllable metal complex loading. The theoretical calculations show that the oxidized units can strongly bond metal complexes while reduced units don't react significantly due to a high formation energy. Both units with proper proportions can cooperatively control the concentration and spatial distribution of metal complexes in the PANI framework. After thermal treatment, the metal/PANI composites are transformed into a series of inorganic-carbon hybrids including metals and metal oxides, carbides, and sulfides. This novel strategy not only significantly improves the catalytic performance of non-noble metal hybrid materials but also greatly increases the utilization efficiency of noble metal catalysts in the hydrogen evolution reaction. Surprisingly, the optimized Pt@NC catalyst exhibits an ultrahigh mass activity that is $\sim 5$.3-times better than the commercial Pt/C catalyst.

Disciplines

Engineering | Physical Sciences and Mathematics

\section{Publication Details}

Wang, Z., Sun, K., Henzie, J., Hao, X., Ide, Y., Takei, T., Bando, Y. \& Yamauchi, Y. (2018). Electrochemically in situ controllable assembly of hierarchically-ordered and integrated inorganic-carbon hybrids for efficient hydrogen evolution. Materials Horizons, 5 (6), 1194-1203.
\end{abstract}

\section{Authors}

Zhongli Wang, Keju Sun, Joel Henzie, Xianfeng Hao, Yusuke Ide, Toshiaki Takei, Yoshio Bando, and Yusuke Yamauchi 


\title{
Electrochemically In-Situ Controllable Assembly of
}

\section{Hierarchically-Ordered and Integrated Inorganic-Carbon Hybrids}

\section{for Efficient Hydrogen Evolution}

\author{
Zhong-Li Wang, ${ }^{1}$ Keju Sun, ${ }^{2}$ Joel Henzie* ${ }^{1}$ Xianfeng Hao, ${ }^{2}$ Yusuke Ide, ${ }^{1}$ Toshiaki Takei, ${ }^{1}$ \\ Yoshio Bando $*^{1,3}$ and Yusuke Yamauchi ${ }^{4}$
}

1 International Center for Materials Nanoarchitectonics (WPI-MANA), National Institute for Materials Science (NIMS), 1-1 Namiki, Tsukuba, Ibaraki 305-0044, Japan

2 Key Laboratory of Applied Chemistry, College of Environmental and Chemical Engineering, Yanshan University, Qinhuangdao, 066004, China

3 Australian Institute for Innovative Materials (AIIM), University of Wollongong, Squires Way, North Wollongong, NSW 2500, Australia

4 School of Chemical Engineering \& Australian Institute for Bioengineering and Nanotechnology (AIBN), The University of Queensland, Brisbane, QLD 4072, Australia

E-mails: HENZIE.Joeladam@nims.go.jp; Bando.Yoshio@nims.go.jp; y.yamauchi@uq.edu.au 


\begin{abstract}
:
Inorganic-carbon hybrid materials are an emerging class of nanostructured catalysts that can enhance various energy-oriented electrochemical reactions. Despite recent progress, it is still very challenging to controllably generate the hybrid carbon architecture and its inorganic components with a single approach. Inspired by the flexible redox properties of conductive polyaniline (PANI) polymer, we develop a redox-units cooperative assembly strategy to synthesize hierarchically-ordered and integrated inorganic-carbon hybrids by electrochemically constructing the nanostructures of PANI and then modifying their redox states to controllably bond different metal complexes. The needle-branched PANI nanofibers are assembled in situ into a three-dimensional (3D) hierarchical framework on carbon paper by an anion induced electrochemical polymerization. Interestingly, tuning the redox states of PANI with a potentiostatic method achieves a controllable metal complex loading. The theoretical calculations show that the oxidized units can strongly bond metal complexes while reduced units hardly react due to high formation energy. Both units with proper proportions can cooperatively control the concentration and spatial distribution of metal complexes in the PANI framework. After thermal treatment, the metal/PANI composites are transformed into a series of inorganic-carbon hybrids including metals and metal oxides, carbides, sulfides. This novel strategy not only significantly improves the catalytic performance of non-noble metal hybrid materials but also greatly increases the utilization efficiency of noble metal catalysts for the hydrogen evolution reaction. Surprisingly, the optimized Pt@NC catalyst exhibits an ultrahigh mass activity that is 25.3-times better than commercial Pt/C catalyst.
\end{abstract}




\section{Introduction}

Creating sustainable alternatives to fossil fuels requires new strategies to harness the energy from renewable resources (i.e. solar, wind, tidal, etc.) and store that energy in chemical bonds ${ }^{1}$. By developing efficient electrocatalysts that can utilize abundant molecules like $\mathrm{H}_{2} \mathrm{O}, \mathrm{CO}_{2}$, and $\mathrm{N}_{2}$, we can generate sustainable fuels and other fine chemicals. Better electrocatalysts are the key to the viability of this technology because they can increase the rate, efficiency, and selectivity of the desired electrochemical reactions ${ }^{2}$. Catalysts made from precious metals (i.e. $\mathrm{Pt}, \mathrm{Rh}, \mathrm{Pd}$, etc.) are ubiquitous in energy related electrochemical reactions, but their scarcity and high cost limit their utility in large scale applications. Catalysts that use more Earth abundant metals are being actively explored, but more work must be done to find materials that perform as well precious metals ${ }^{3}$. Therefore, it is necessary to develop flexible strategies to generate new electrocatalytic materials and rapidly access their electrocatalytic performance.

The inorganic-carbon hybrid materials are hierarchically ordered arrangements of ultrafine metal and metal composite (oxides, sulfides, etc) nanoparticles on tailored carbon architectures ${ }^{4-6}$. Strong bonding interactions between the metal and carbon substrate improves the electrical conductivity, structure stability, and potentially generates synergistic catalytic sites. Typically, the full range of hybrid inorganic-carbon catalysts (e.g., metal oxides, sulfides, carbides, nitrides, phosphides) are synthesized via two main strategies: (1) functionalizing nanocarbon such as graphene $e^{7-9}$, carbon nanotubes ${ }^{10-14}$ and active carbons ${ }^{15-17}$ and growing inorganic nanomaterials on them. This is a multistep process that limits where both carbon and inorganic nanostructures are synthesized separately and then combined. And (2) thermal treatment of assembled organic and inorganic precursors. In this case, both components are produced simultaneously during thermal treatment, which simplifies the preparation of hybrid materials. So far, the limiting step of the thermal method is obtaining sufficient variety metal-organic composite precursors. Numerous approaches have been developed to prepare these composite precursors, including homogeneously mixing metal salts and organic molecules (e.g., dicyandiamide, melamine, ionic liquid) ${ }^{18-21}$ or polymers (e.g., polydiaminopyridine $)^{22,23}$, complexing metal ions with organic ligands (e.g., ethylenediaminetetraacetate anions $\left(\mathrm{EDTA}^{4-}\right)^{24}$, phytic acid $\left.{ }^{25,26}\right)$, and embedding metal ions in metal-organic frameworks (MOFs) ${ }^{27-33}$. However, most of these approaches use weak chemical interactions between the metal and organic precursors, which make it difficult to control the components. Moreover, the precursors have irregular 
morphologies that result in disordered structures. Disorder in the context of electrocatalysis is undesirable because it can cause serious aggregation, leading to sluggish mass diffusion. Therefore, it is necessary to develop flexible strategies to controllably generate the hybrid carbon architecture and its inorganic components with a single approach.

PANI is a conductive polymer with good environmental stability, a high level of processability, and supports interesting redox properties associated with the chain heteroatom of $\mathrm{N}^{34-36}$. PANI can form a variety of nanostructures by adjusting the conditions of polymerization. And their unique morphologies are typically well preserved after carbonization, providing an effective approach to prepare carbon materials with a desired morphology. Moreover, the intrinsic redox states of PANI (Fig. 1a) can vary from that of the fully oxidized pernigraniline (PNA, $\mathrm{y}=0$, all oxidized units) to fully reduced leucoemeraldine (LM, $\mathrm{y}=1$, all reduced units). The $50 \%$ intrinsically oxidized PANI is called emeraldine (EM, y $=0.5$, half oxidized units and half reduced units). ${ }^{34}$ The different redox states of PANI provide specific electronic structures that could promote different levels of bonding between the polymer and metal complexes. Fig. 1b shows density-functional-theory (DFT) calculations of the three idealized redox states of PANI. The fully oxidized PNA state has a remarkably lower conduction band edge compared to the fully reduced LM state. In addition, Fig. 1c shows the conduction band edge consists of some bonding orbitals on PNA, while it is composed by anti-bonding orbitals on LM. Clearly, the oxidized PNA has the stronger capability to accept and stabilize the negatively charged metal complexes compared to the reduced LM state. We hypothesized that this phenomena would promote the formation of a strongly coupled metal-polymer framework, which could be generated prior to carbonization to create inorganic-carbon materials with well-dispersed metal nanocrystals.

Here we successfully develop a redox-units cooperative assembly strategy to make a range of 3D inorganic-carbon hybrids by electrochemically constructing the nanostructures and then modifying their redox states of PANI to controllably bond different metal complexes. The needle-branched PANI nanofibers are assembled in situ into a 3D hierarchical framework on conductive carbon paper by an anion induced electrochemical polymerization. Then, the redox states of PANI are tuned via a potentiostatic method to infiltrate the structure with metal complexes. As expected, the loading of metal complexes exhibited a regular change with the varying of redox states of the PANI polymer. DFT calculations further demonstrate 
that the oxidized units can strongly bond metal complex ions while reduced units hardly react due to high formation energy. Both units can synergistically control the concentration and spatial distribution of metal complex ions in the PANI framework. After thermal treatment, the metal/PANI composite precursors were transformed into a 3D network of self-supported hybrid catalysts on an N-doped carbon framework. And since these catalysts are grown on a current collector they can be directly used as the working electrode in the catalytic reaction. The hierarchical structures provide continuous mass transport pathways with minimal diffusion resistance, yielding high electrocatalytic kinetics. This method is highly flexible so we can prepare a variety of hybrid catalysts composed of metals on N-doped carbon (M@NC) including $\mathrm{Co} @ \mathrm{NC}, \mathrm{Ni} @ \mathrm{NC}$, $\mathrm{Fe}_{3} \mathrm{O}_{4} / \mathrm{Fe}_{3} \mathrm{C} @ \mathrm{NC}$, amorphous $\mathrm{MoC}_{\mathrm{x}} \mathrm{S}_{\mathrm{y}} @ \mathrm{NC}, \mathrm{MoC} @ \mathrm{NC}$, and Pt@NC. Catalytic HER is chosen as the model reaction, and the activities of non-noble Co@NC and noble Pt@NC are investigated. The optimized Co@NC catalyst exhibits a low overpotential of $145 \mathrm{mV}$ at $10 \mathrm{~mA} \mathrm{~cm}^{-2}$ and considerable stability in alkaline media, which is much better than most $\mathrm{Co} / \mathrm{C}$ materials synthesized by traditional methods. The optimized Pt@NC catalyst exhibits an ultrahigh mass activity that is 5.3-times better than commercial Pt/C catalyst. The highly dispersed Pt nanoclusters in the hierarchically-ordered and integrated carbon structure expose abundant active sites and enable more efficient utilization of precious metals. 


\section{Results and Discussion}

The in situ electrochemical polymerization of PANI was carried out on carbon paper by galvanostatic method at a current density of $2 \mathrm{~mA} \mathrm{~cm}^{-2}$ for $4000 \mathrm{~s}$ (Fig. S1a). The morphologies of the PANI are shown in Fig. 2a and Fig. S1b-c. The PANI nanofibers are composed of interconnected, branched needles that form a 3D porous hierarchical structure. Interestingly, the fine structure of the branched-needles depends on the type of acid added to the electrolyte. As shown in Fig. S1d-f, the lengths of the branched needles of PANI synthesized using electrolyte and $\mathrm{HCl}, \mathrm{H}_{2} \mathrm{SO}_{4}$, and $\mathrm{H}_{3} \mathrm{PO}_{4}$ were obviously shorter compared to $\mathrm{HNO}_{3}$, especially for $\mathrm{H}_{3} \mathrm{PO}_{4}$-based PANI, which had almost no branched structures. The four type acids have the same cation but different anions. The anions produce different nanostructures of PANI, which is a typical anionic effect ${ }^{37-39}$. The anions form ion pairs with anilinium ions, and the stability of these ion pairs determines the kinetics of the polymerization reaction. This is because the dissociation of the ion pair precedes the electron transfer reaction, which affects the rate of polymer growth and results in the different morphologies. As reflected by the V-t curves for all four acids (Fig. S1a) the highest potential was needed to drive the electropolymerization of PANI in electrolyte with $\mathrm{HNO}_{3}$. This indicates that the nitrate and anilinium ion pair is the most stable. The slow kinetic of the polymerization reaction in $\mathrm{HNO}_{3}$ electrolyte could provide opportunities to construct hierarchical structures. Furthermore, it is generally assumed that there are two parallel nucleation and growth processes in the polymerization reaction. One is direct monomer oxidation on the substrate and the other is the autocatalytic oxidation that takes place between the electrochemically generated PNA and the monomer ${ }^{37}$. The high potential in $\mathrm{HNO}_{3}$ electrolyte may produce more PNA units, which would induce instantaneous nucleation and 3D growth, resulting in branched needle structures.

The electrochemical redox behavior of the PANI polymer was evaluated with cyclic voltammetry (CV) in a $0.5 \mathrm{M} \mathrm{HNO}_{3}$ electrolyte using a three-electrode system (Fig. 2b). Two redox peaks that were observed in the CV curve are attributed to LM/EM and EM/PNA transitions in the PANI polymer. According to the CV curve, four potentials are selected to tailor the redox states by the potentiostatic method (Fig. 2c). The samples are treated at selected potentials for $0.5 \mathrm{~h}$. Interestingly, in their FTIR spectra (Fig. 2d) the relative strength of two peaks at 1580 and $1493 \mathrm{~cm}^{-1}$, which correspond to quinonid- and benzenoid-ring stretching deformations $\mathrm{s}^{35}$, respectively, varies with the applied potentials from $-0.5 \mathrm{~V}$ to $0.8 \mathrm{~V}$, accompanying the 
change of color from light yellow to green and then dark brown (Fig. S2). At low potential (-0.5V), the FTIR spectrum exhibits a very low intensity ratio of the $1580 \mathrm{~cm}^{-1}: 1493 \mathrm{~cm}^{-1}$ peaks, suggesting the presence of mostly benzenoid units. At middle potential $(0.45 \mathrm{~V})$, nearly equal intensities for both peaks are observed, which indicates there are equal amounts of benzenoid and quinonoid units. At high potential (0.8 V), the intensity of the quinonoid to benzenoid band ratio is enhanced. This result indicates that PANI changes from reduced state at low potential to oxidized sate at high potential. During treating in acid condition, the doping of proton acid will proceed simultaneously, in which $\mathrm{H}^{+}$ions are bonded to amine or imine groups and $\mathrm{NO}_{3}{ }^{-}$anions will also be embedded between polymer chains to maintain neutral electricity. The values of Zeta potentials are of $38.82,39.13,39.96$, and $38.81 \mathrm{mV}$ for $-0.5,0,0.45$ and $0.8 \mathrm{~V}$ treated PANI, respectively. It means PANIs are positively charged, which provide the possibility to adsorb negatively charged metal complexes by anion exchange and electrostatic interaction. Interestingly, the adsorption capacities of each metal complex varied with the redox states of PANI (Fig. 2e, f). The adsorption capacities in oxidized PANIs are higher than that in reduced ones, which supports our hypothesis that the imine groups in the oxidized PNA state interacts more strongly with metal complexes. After binding metal complexes, the characteristic peaks of the ligands are observed by FTIR in the PANI polymer hybrids (Fig. S3). According to the HER performance, PANI-Co(CN) 6 and PANI-Pt $(\mathrm{CN})_{4}$ hybrid systems well reflect the advantages of the synthesis strategy, the former represents non-noble metal catalysts and the latter represents the noble metal catalysts. So they are selected as two examples to systematically investigate the structure, composition and HER catalytic performance.

The interactions between the redox units of PANI and Co or Pt complex ions are first studied by the DFT method. As shown in Fig 3, the formation energies of PNA-Co and PNA-Pt hybrids are -2.22 and -2.28 $\mathrm{eV}$, respectively, indicating a spontaneous reaction, while for LM-Co and LM-Pt hybrids, in contrast, the energy are +1.67 and $+1.66 \mathrm{eV}$, respectively, indicating a difficult reaction. This result clearly demonstrates oxidized unis can easily react with metal complex ions to form stable PANI-metal hybrids by ion bonding, but reduced units are very difficult to attract the ions due to high energy, which confirms our hypothesis derived from the electronic structure of PANI. That is to say, there is an interesting selective ion-bonding effect in the redox units of the PAni polymer that oxidized units can bond to metal complexes and reduced units cannot. Moreover, except that, there is another unexpected phenomenon. For the mixed units of EM, 
the formation enegies are -2.39 and $-2.46 \mathrm{eV}$ for EM-Co and EM-Pt hybrids, respectively, which is a little lower than pure oxidized units of PNA. This means both units coexist can synergisticly improve the interation between PANI and metal complex ions. The change trend from LM to PNA is in consistent with our experiments in Fig 2f. Although the reduced units are difficult to bind with metal complex ions, they act as spacers between oxidized units and indirectly affect the spatial distribution of the oxidized units. The highly dispersed oxidized units will be easier to bond metal complex ions. By virtue of the strong bonding energy, the oxidized units control the concentration of the metal complex ions tethered to the PANI polymer while the reduced units indirectly control the spatial distribution of the ions. Therefore, electrochemically tuning the relative concentrations of both units will enable the controllably assembly of metal complex ions in the PANI frameworks.

By using the above complexed metal/PANI composites as precursors, thermal treatment created a variety of hybrid catalysts (Fig. 4a). Since the applied potential on PANI determines the composition of the hybrids, samples are called M@NC(x), where $x$ indicates the applied potential. From the SEM images (Fig. 4b,c and Fig. S4), it can be seen that the morphologies of 3D branched PANI fibers are well-preserved after thermolysis for all Co@NC samples. In the TEM images of Co@NC(0.45) (Fig. 4d and Fig. S5), Co nanoparticles are clearly observed with average sizes of $20 \mathrm{~nm}$. High-resolution TEM (HRTEM) image of Co@NC(0.45) (Fig. 4e) show that Co nanoparticles were wrapped in carbon nanolayers. In addition, some crystalline graphite layers with a lattice spacing of ca. $0.34 \mathrm{~nm}$ are observed, which indicate Co nanoparticles catalyze the graphitization of amorphous carbon around the nanoparticles. The energy-dispersive X-ray (EDX) spectrum analysis shows that the Co metal atom is homogeneously distributed over the entire carbon fibers (Fig. 4f-i). This indirectly demonstrates that the metal complexes are uniformly bonded to PANI at different redox states. The $\mathrm{N}_{2}$ sorption isotherm exhibits mixed type I and IV isotherms, indicates the presence of micropores and mesopores (Fig. S5). The specific surface area is $293 \mathrm{~m}^{2}$ $\mathrm{g}^{-1}$ for Co@NC(0.45). For comparison, Co@NC(-0.2) is aslo characterized (Fig. S5). The powder X-ray diffraction (XRD) patterns (Fig. 5a) show that there was an intense diffraction peaks at $\sim 44^{\circ}$ in Co@NC(0.45), which matches the (111) plane of face-centered-cubic (fcc) Co nanoparticles. Since the Co loadings in the precursor first increase and then decrease from Co@NC(-0.5) to Co@NC(0.8) (Fig. 2f), the peak intensities of metal Co in the carbonized products show a similar change trend in the XRD (Fig. 5a) 
and Raman spectra (Fig. 5b). ICP results show that the concentrations of Co@NC(-0.5, -0.2, 0, 0.45, and $0.8)$ are $0.7,1.2,4.6,6.7$, and $4.6 \mathrm{wt} \%$, respectively. The composition of samples is further identified by X-ray photoelectron spectroscopy (XPS). As shown in Fig. 5c, the two core-level signals located at $\sim 780$ and $796 \mathrm{eV}$ are attributed to $\mathrm{Co} 2 \mathrm{p} 3 / 2$ and Co2p1/2, respectively. After deconvolution, the peak around 779.9 $\mathrm{eV}$ is assigned to $\mathrm{Co}^{0}$, while that around $782.1 \mathrm{eV}$ corresponds to $\mathrm{CoO}$ phase. On the surface, it is inevitable for a small amount of Co metal to be oxidized. For the $\mathrm{N}$ 1s spectra (Fig. 5d), three peaks are fitted at binding energy of 398.6, 400.8, and $402.3 \mathrm{eV}$, corresponding to the pyridinic $\mathrm{N}$, graphitic $\mathrm{N}$ and oxidized $\mathrm{N}$, respectively. All of these observations show that varying the redox states of PANI polymer enables the synthesis of $\mathrm{Co} / \mathrm{N}$-doped carbon hybrids with different compositions.

The electrocatalytic activity of Co@NC for HER was evaluated in $1 \mathrm{M} \mathrm{KOH}$ solution using a typical three electrode system. To access the performance of the catalyst we compared pure carbon support (NC) and commercial PANI derived $\mathrm{Co} / \mathrm{NC}(\mathrm{COM} \mathrm{Co} / \mathrm{NC})$ under the same conditions. Fig. 6a shows the polarization curves of samples prepared by applying different potentials on PANI from $-0.5 \mathrm{~V}$ to $0.8 \mathrm{~V}$. Interestingly, NC derived from pure PANI has low activity, but it becomes highly active after incorporation of Co metal. This observation demonstrates the crucial role of the Co species in HER. From the series of samples, it can be seen that the HER activities first increases and then decreases in the overpotential and reduction current with increasing applied potentials from $-0.5 \mathrm{~V}$ to $0.8 \mathrm{~V}$, which is consistent with the change of the Co content (Fig. 6b). Among those samples, Co@NC(0.45) exhibited the best activity. The overpotentials required to drive cathodic current densities of 10,20, and $100 \mathrm{~mA} \mathrm{~cm}^{-2}$ are 145, 180, and 254 $\mathrm{mV}$, respectively. These overpotentials are much lower than $\mathrm{COM} \mathrm{Co} / \mathrm{NC}$, and at $10 \mathrm{~mA} \mathrm{~cm}^{-2}$, its overpotential exceeds $350 \mathrm{mV}$. Moreover, the acticities of $\mathrm{Co} @ \mathrm{NC}(0.45)$ are also better than most $\mathrm{Co} / \mathrm{C}$ based catalysts reported in the literature in alkaline media (Table S1). Combined with the results of TEM and XRD, it might be logical to assume that the excellent activity in $\mathrm{Co} @ \mathrm{NC}(0.45)$ originates from the carbon-encapsulated Co and exposed Co nanoparticles on the surface. However, pure Co particles have low activity (Fig. S6). This indicates that N-doped carbon support also plays an important role in catalysis. Besides the intrinsic high conductivity, the large surface area of branched carbon fibers prevents the accumulation of metal particles and facilitates their uniform distribution, resulting in more active catalytic sites. Compared with the structures of COM Co/NC (Fig. S7), it is found that the irregular commercial PANI 
particles are seriously aggregated into large particles after carbonization, which highlights the super stalility of our optimized PANI nanostructures. Therefore, the improved activity of Co@NC(0.45) is the result of a synergistic effect between the Co metal catalyst and the 3D hierarchical $\mathrm{N}$-doped carbon architecture. The Co@NC(0.45) also exhibits a moderate Tafel slope of $103 \mathrm{mV} \mathrm{dec}{ }^{-1}$ (Fig. 6c), which suggests that HER occurs through a Volmer-Heyrovsky mechanism. The durability of $\mathrm{Co} @ \mathrm{NC}(0.45)$ catalyst was examined by chronopotentiometric measurement. The catalyst maintains a stable overpotential for $24 \mathrm{~h}$ at an current

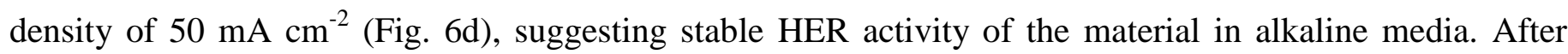
10,000 cycles, the polarization curve decrease only slightly (Fig. S6). Moreover, after long-term tests, the nanostructures of catalyst are well retained (Fig. S8). Except Co@NC, other non-noble hybrid catalysts including $\mathrm{Ni} @ \mathrm{NC}, \mathrm{Fe}_{3} \mathrm{O}_{4} / \mathrm{Fe}_{3} \mathrm{C} @ \mathrm{NC}, \mathrm{MoC}_{\mathrm{x}} \mathrm{S}_{\mathrm{y}} @ \mathrm{NC}$, and MoC@NC were also prepared by this synthesis strategy and exhibited good HER activity, especially $\mathrm{Ni@NC}(0.45)$, with performance close to the Co@NC(0.45) samples (Fig. S9-14).

The cooperative assembly strategy not only improves the performance of non-noble metal hybrid materials but also increases the utilization efficiency of noble metal catalysts. Using this strategy, we prepared a series of Pt@NC catalysts. The morphologies of the carbonized fibers maintain the original hierarchical structure of needle-branched features after carbonization (Fig. 7a) and the fibers are uniformly covered with highly dispersed Pt nanoclusters with an average diameter $1.8 \pm 0.5 \mathrm{~nm}$ (Fig. 7b). The HRTEM image shows the crystal structure of Pt nanoclusters (Fig. 7c) and the corresponding fast-Fourier transform (FFT) (inset of Fig. 7c) matches the electron diffraction pattern of a cubic Pt (fcc) lattice. The spacing of the crystal lattices are 0.196 and $0.226 \mathrm{~nm}$, which can be attributed to the (200) and (111) atomic planes. EDX spectrum show all elements including $\mathrm{Pt}, \mathrm{C}$, and $\mathrm{N}$ are homogeneously distributed throughout the carbon fibers (Fig. 7d-g). The structure of the samples prepared at other potentials was also investigated with HRTEM (Fig. S15, 16). The density of Pt nanoclusters clearly increased from Pt@NC(-0.5) to Pt@NC(0.45), with the largest observed diameters of $\sim 2.5 \mathrm{~nm}$ in Pt@ NC(0.45). The XRD diffraction peaks of Pt first increase and then decrease, similar with Co@NC samples (Fig. S16a). The concentrations of Pt@NC(-0.5, $-0.2,0,0.45$, and 0.8$)$ are $5.5,9.0,19.7,25.0$, and $20.2 \mathrm{wt} \%$, respectively, indicating the regularly change with the applied potentials on PANI. 
The electrocatalytic HER performance of Pt@NC samples are also conducted in $1 \mathrm{M} \mathrm{KOH}$ solution. $\mathrm{NC}$ and commercial $20 \mathrm{wt} \% \mathrm{Pt} / \mathrm{C}(\mathrm{COM} 20 \% \mathrm{Pt} / \mathrm{C})$ are used for comparison. Fig. 8a shows the polarization curves of NC versus a series of Pt@NC samples. The performance of the NC sample is negligible compared to the Pt-containing catalysts, proving that performance is primarily caused by the Pt nanoclusters. For Pt@NC samples, the change of performance is very interesting. From Pt@NC(-0.5) to Pt@NC(-0.2), the activity is greatly improved, while from Pt@ $\mathrm{NC}(-0.2)$ to $\mathrm{Pt} @ \mathrm{NC}(0.8)$, the performance change little. This means the activity of 9.0\% Pt in Pt@NC(-0.2) reaches the level of 25.0\% Pt in Pt@ NC(0.45), which indicates the super utilization efficiency of Pt in Pt@ $\mathrm{NC}(-0.2)$. In $\mathrm{Pt} @ \mathrm{NC}(0.45)$, the increased particle size, particle aggregation, and possible carbon coverage lead to the low efficiency of Pt at high loading. In contrast, in Pt@NC(-0.2) the optimized loading and highly dispersion of Pt nanoclusters combining with the hierarchically-ordered and integrated carbon structure expose abundant active sites and enable more efficient utilization of precious metals. As a result, with only 9.0 wt\% loading $\mathrm{Pt} @ \mathrm{NC}(-0.2)$ requires very small overpotentials of 28,42 , and $61 \mathrm{mV}$ to achieve current densities of 10,20 , and $100 \mathrm{~mA} \mathrm{~cm}{ }^{-2}$, respectively. These overpotentials are better than COM 20\% Pt/C and even much better than other previously reported Pt-based HER catalysts (Table S2). Compared to the serious aggregation in the morphologies of COM $20 \%$ Pt/C and comercial PANI derived Pt/C (Fig. S17), the unique advantages of the hierarchical nature of our integrated electrode are full reflected by the super activities. It is noted that the above mentioned activities are all based on electrode's geometrical area. For noble metal catalysts, mass activity also plays an important role in the large-scale applications. ${ }^{40,41}$ Fig. $8 \mathrm{c}$ and $8 \mathrm{~d}$ show the comparisons of mass activities of the Pt@NC(x) samples and COM 20\% Pt/C based on the Pt mass. Surprisingly, Pt@NC(-0.2) delivered a mass activity of $1.07 \mathrm{~A} \mathrm{mg}_{\mathrm{Pt}}{ }^{-1}$ at the overpotential of $50 \mathrm{mV}$, which is 5.3 times higher than commercial $\mathrm{Pt} / \mathrm{C}$. Additionally, Pt@NC(-0.2) has a small Tafel slope of $28 \mathrm{mV}$ decade ${ }^{-1}$, which is $16 \mathrm{mV}$ decade $\mathrm{e}^{-1}$ lower than that of COM 20\% Pt/C (Fig. 8e). Finally, chronopotentiometric measurements show that Pt@NC(-0.2) exhibits excellent durability (Fig. 8f). After 10,000 CV cycles, the polarization curve of Pt@NC(-0.2) shows negligible degradation (Fig. S18). Moreover, the Ru nanostructures are well maintained after long-time test, indicating the high stability of the catalyst (Fig. S19). 


\section{Conclusions}

We demonstrate a redox-units cooperative assembly strategy to in situ synthesize inorganic-carbon hybrid catalysts by electrochemically tuning the 3D morphologies and redox states of PANI. This is a holistic approach to generate inorganic-carbon integrated electrodes with superior material utilization efficiency. The selective ion-bonding effect in the redox units of PANI provides a means of regulating assembly behavior of the metal complex ions. Electrochemically tailoring the redox states of PANI can not only adjust the loading amount of inorganic components but also control their distribution on the carbon support. By using this strategy, a variety of inorganic-carbon hybrids with controllable structures and components have been prepared including metals and metal oxides, carbides, sulfides. As one of the potential applications, the hybrids exhibit high activities and stabilities for HER and the optimized Pt@NC catalyst exhibits an ultrahigh mass activity that is $\sim 5.3$-times better than commercial Pt/C catalyst. This synthesis strategy could also be extended to prepare other advanced hybrid materials on various electrodes for energy related electrochemical devices such as fuel cells and lithium batteries.

\section{Acknowledgment}

This research was partially supported by the Grant-in-Aid for International Research Fellow of the Japan Society for the Promotion of Science (JSPS, Project 15766). 


\section{References}

1 Z. W. Seh, J. Kibsgaard, C. F. Dickens, I. Chorkendorff, J. K. Nørskov, T. F. Jaramillo, Science, 2017, 355, eaad4998.

2 S. Gao, Y. Lin, X. Jiao, Y. Sun, Q. Luo, W. Zhang, D. Li, J. Yang, Y. Xie, Nature, 2016, 529, 68-72.

3 Y. Y. Liang, Y. G. Li, H. L. Wang, H. J. Dai, J. Am. Chem. Soc., 2013, 135, 2013-2036.

4 J. Wang, F. Xu, H. Jin, Y. Chen, Y. Wang, Adv. Mater., 2017, 29, 1605838

5 Y. Hao, Y. Xu, W. Liu, X. Sun, Mater. Horiz., 2018, 5, 108-115.

6 X. Xiao, L. Tao, M. Li, X. Lv, D. Huang, X. Jiang, H. Pan, M. Wang, Y. Shen, Chem. Sci., 2018, 9, 1970-1975.

7 W. J. Zhou, J. Zhou, Y. C. Zhou, J. Lu, K. Zhou, L. J. Yang, Z. H. Tang, L. G. Li, S. W. Chen, Chem. Mater., 2015, 27, 2026-2032.

8 S. Peng, L. Li, X. Han, W. Sun, M. Srinivasan, S. G. Mhaisalkar, F. Cheng, Q. Yan, J. Chen, S. Ramakrishna, Angew. Chem. Int. Ed., 2014, 53, 12594-12599.

9 J. J. Duan, S. Chen, B. A. Chambers, G. G. Andersson, S. Z. Qiao, Adv. Mater., 2015, 27, 4234-4241.

10 M. Tavakkoli, T. Kallio, O. Reynaud, A. G. Nasibulin, C. Johans, J. Sainio, H. Jiang, E. I. Kauppinen, K. Laasonen, Angew. Chem. Int. Ed., 2015, 54, 4535-4538.

11 D. Y. Wang, M. Gong, H. L. Chou, C. J. Pan, H. A. Chen, Y. P. Wu, M. C. Lin, M. Y. Guan, J. Yang, C. W. Chen, Y. L. Wang, B. J. Hwang, C. C. Chen, H. J. Dai, J. Am. Chem. Soc., 2015, 137, 1587-1592.

12 D. J. Li, U. N. Maiti, J. Lim, D. S. Choi, W. J. Lee, Y. Oh, G. Y. Lee, S. O. Kim, Nano Lett., 2014, 14, 1228-1233.

13 D. McAteer, Z. Gholamvand, N. McEvoy, A. Harvey, E. O’Malley, G. S. Duesberg, J. N. Coleman, ACS Nano, 2016, 10, 672-683.

14 Q. Liu, J. Q. Tian, W. Cui, P. Jiang, N. Y. Cheng, A. M. Asiri, X. P. Sun, Angew .Chem. Int. Ed., 2014, 53, 6710-6714.

15 J. Wang, H. X. Zhong, Z. L. Wang, F. L. Meng, X. B. Zhang, ACS Nano, 2016, 10, 2342-2348.

16 X. X. Zou, X. X. Huang, A. Goswami, R. Silva, B. R. Sathe, E. Mikmekov, T. Asefa, Angew. Chem. Int. Ed., 2014, 53, 4372-4376.

17 Z. Y. Yu, Y. Duan, M. R. Gao, C. C. Lang, Y. R. Zheng, S. H. Yu, Chem. Sci., 2017, 8, 968-973.

18 H. Y. Jin, J. Wang, D. F. Su, Z. Z. Wei, Z. F. Pang, Y. Wang, J. Am. Chem. Soc., 2015, 137, 2688-2694.

19 Y. P. Liu, G. T. Yu, G. D. Li, Y. H. Sun, T. Asefa, W. Chen, X. X. Zou, Angew .Chem. Int. Ed., 2015, 54, 10752-10757.

20 S. P. Wang, J. Wang, M. L. Zhu, X. B. Bao, B. Y. Xiao, D. F. Su, H. R. Li, Y. Wang, J. Am. Chem. Soc., 2015, 137, 15753-15759.

21 Zhu, J. X. K. Sakaushi, G. Clavel, M. Shalom, M. Antonietti, T. P. A Fellinger, J. Am. Chem. Soc., 2015, 137, 5480-5485.

22 Y. Zhao, K. Kamiya, K. Hashimoto, S. Nakanishi, Angew. Chem. Int. Ed., 2013, 52, 13638-13641.

23 Y. Zhao, K. Kamiya, K. Hashimoto, S. Nakanishi, J. Am. Chem. Soc., 2015, 137, 110-113. 
24 J. Deng, P.J. Ren, D. H. Deng, X. H. Bao, Angew. Chem. Int. Ed., 2015, 54, 2100-2104.

25 M. H. Zhuang, X. W. Ou, Y. B. Dou, L. L. Zhang, Q. C. Zhang, R. Z. Wu, Y. Ding, M. H. Shao, Z. T. Luo, Nano Lett., 2016, 16, 4691-4698.

26 G. Zhang, G. C. Wang, Y. Liu, H. J. Liu, J. H. Qu, J. H. Li, J. Am. Chem. Soc., 2016, 138, 14686-14693.

27 H. B. Wu, B. Y. Xia, L. Yu, X. Y. Yu, X. W. Lou, Nat. Commun., 2015, 6, 6512-6519.

28 H. M. Barkholtz, D. J. Liu, Mater. Horiz., 2017, 4, 20-37.

29 Y. J. Tang, M. R. Gao, C. H. Liu, S. L. Li, H. L. Jiang, Y. Q. Lan, M. Han, S. H. Yu, Angew. Chem. Int. Ed., 2015, 54, 12928-12932.

30 B. You, N. Jiang, M. L. Sheng, S. Gul, J. Yano, Y. J. Sun, Chem. Mater., 2015, 27, 7636-7642.

31 Z. Song, W. Liu, N. Cheng, M. N. Banis, X. Li, Q. Sun, B. Xiao, Y. Liu, A. Lushington, R. Li, L. Liu, X. Sun, Mater. Horiz., 2017, 4, 900-907.

32 Y. T. Xu, X. F. Xiao, Z. M. Ye, S. L. Zhao, R. A. Shen, C. T. He, J. P. Zhang, Y. D. Li, X. M. Chen, J. Am. Chem. Soc., 2017, 139, 5285-5288.

33 L. Jiao, Y. X. Zhou, H. L. Jiang, Chem. Sci., 2016, 7, 1690-1695

34 G. Ćirić-Marjanović, Synth. Met., 2013, 177, 1-47.

35 E. T. Kang, K. G. Neoh, K. L. Tan, Prog. Polym. Sci., 1998, 23, 211-324.

36 L. Pan, G. Yu, D. Zhai, H. R. Lee, W. Zhao, N. Liu, H. Wang, B. C. K. Tee, Y. Shi, Y. Cui, Z. Bao, PNAS, 2012, 109, 9287-9292.

37 R. Crdova, M. A. D. Valle, A. Arratia, H. Gmez, J. Electroanal. Chem., 1994, 377, 75-83.

38 S. J. Choi, S. M. Park, J. Electrochem. Soc., 2002, 149, 26-34.

39 Y. Wang, H. D. Tran, L. Liao, X. F. Duan, R. B. Kaner, J. Am. Chem. Soc., 2010, 132, 10365-10373.

40 Z. L. Wang, K. J. Sun, J. Henzie, X. F. Hao, C. Li, T. Takei, Y. M. Kang, Y. Yamauchi, Angew. Chem. Int. Ed., 2018, 57, 5848-5852.

41 S. T. Hunt, M. Milina, A. C. Alba-Rubio, C. H. Hendon, J. A. Dumesic, Y. Román-Leshkov, Science, 2016, 352, 974-978. 
(a)
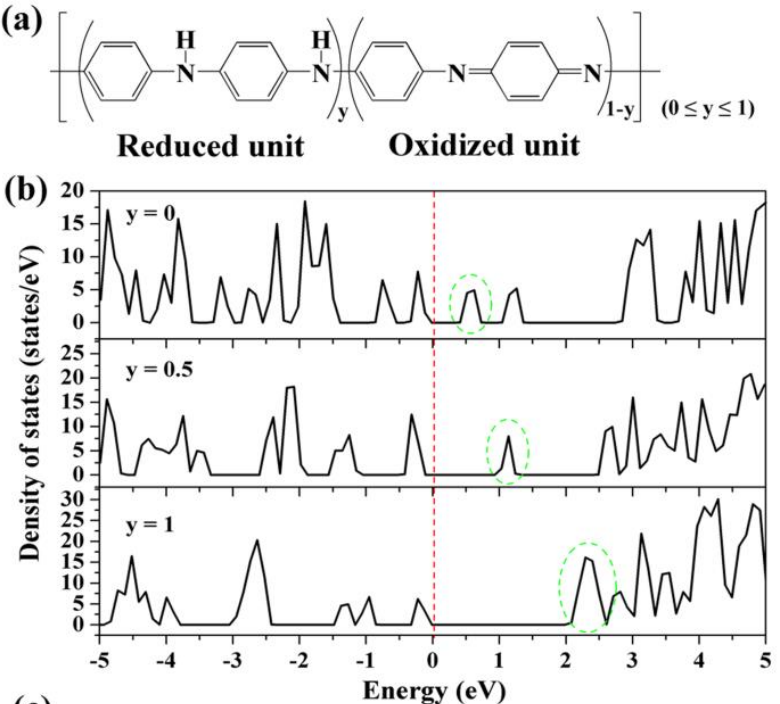

(c)

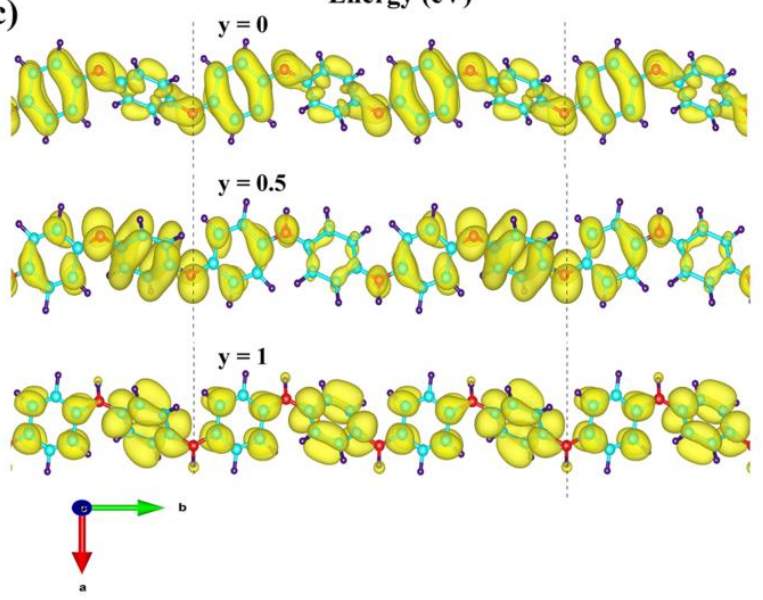

Fig. 1 (a) Molecular structure of PANI. (b) Density of state (DOS) of three redox states of PANI: fully oxidized PNA $(\mathrm{y}=0), 50 \%$ intrinsically oxidized EM $(\mathrm{y}=0.5)$, and fully reduced LM $(\mathrm{y}=1)$. (c) The partial charge density of the lowest unoccupied molecular orbital (LUMO) as marked with a green circle in (b) for three redox states. The cyan, navy and red balls are $\mathrm{C}, \mathrm{H}$, and $\mathrm{N}$ atoms, respectively. The yellow refers to charge density contour with the isovalue of $0.001 \mathrm{e} / \AA^{3}$. 
(a)

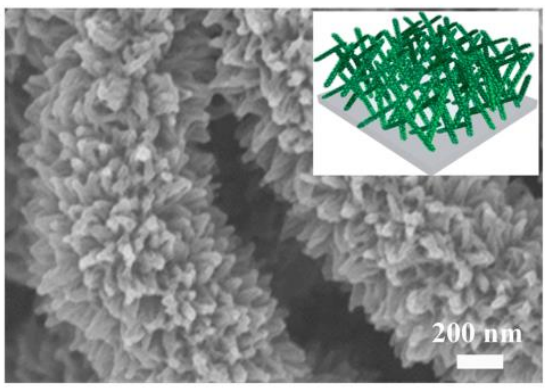

(c)

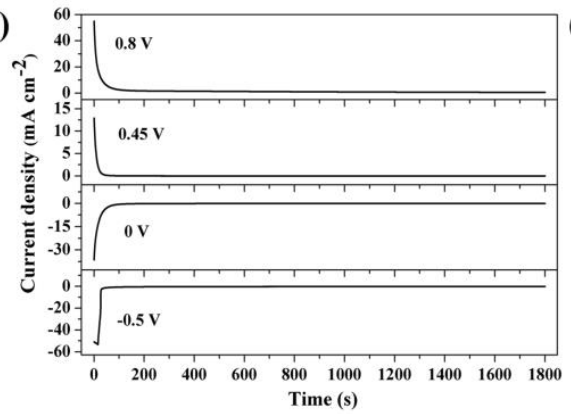

(e)

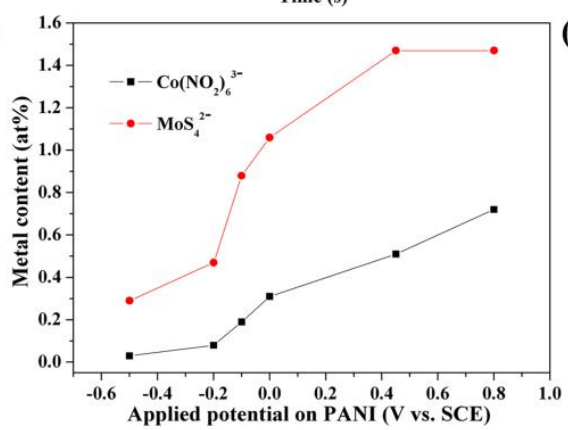

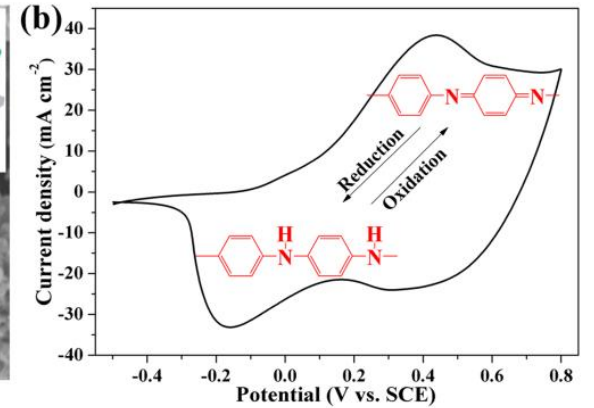

(d)
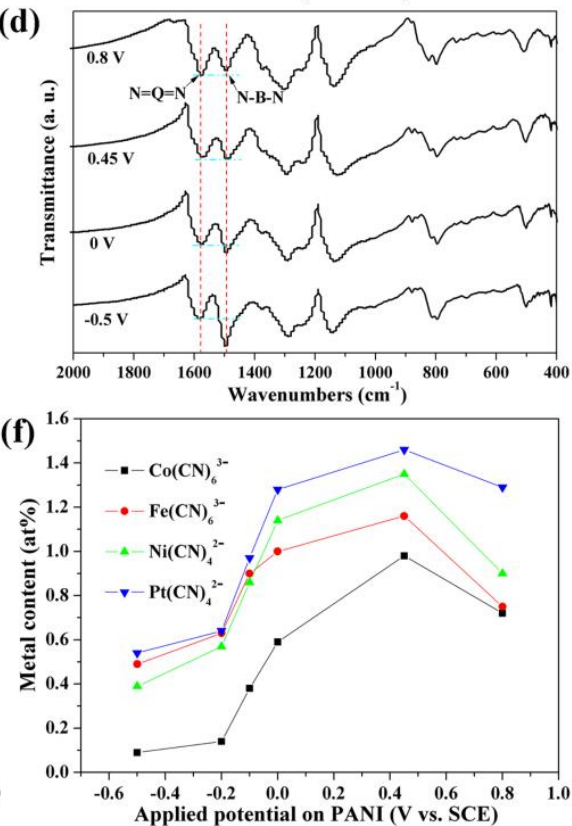

Fig. 2 (a) SEM image of as-prepared PANI. Inset is a hierarchically-ordered and integrated structure model of PANI. (b) $\mathrm{CV}$ curve of the PANI in $0.5 \mathrm{M} \mathrm{HNO}_{3}$ electrolyte. The oxidized quinonoid imine and reduced benzenoid amine units can be reversibly transformed via an external applied potential. (c) i-t curves with four potentials applied on PANI in $0.5 \mathrm{M} \mathrm{HNO}_{3}$ electrolyte. (d) FTIR spectra of four potentials treated PANI. (e) and (f) The adsorption capacities of different metal complexes as a function of applied potentials on PANI. 
(a)
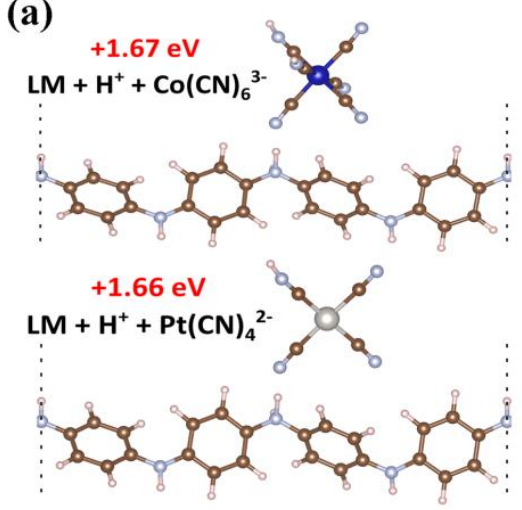

(c)
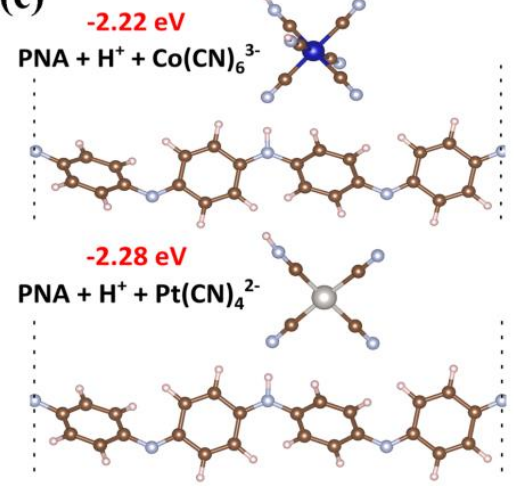

(b)
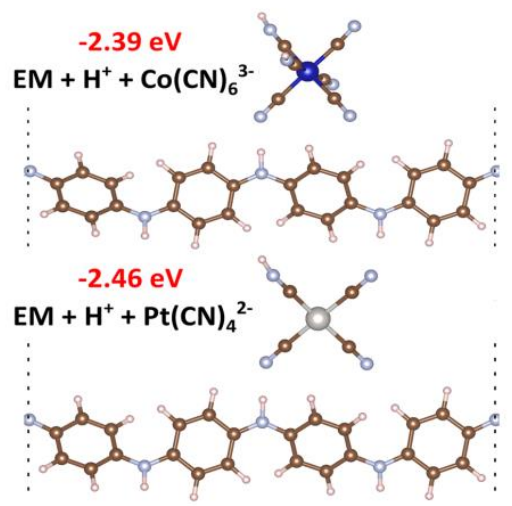

(d)

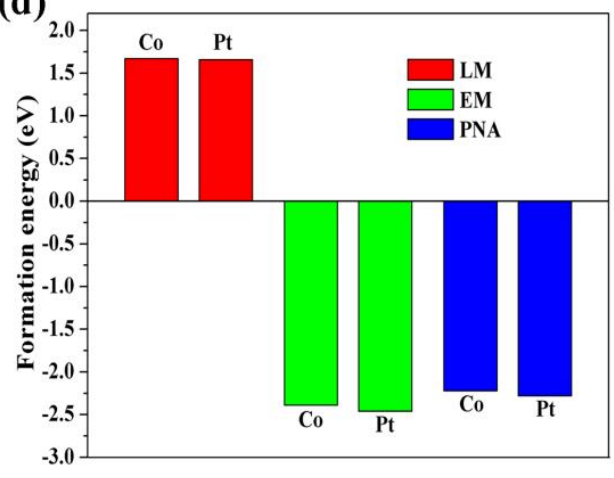

Fig. 3 Structure models and formation energies of PANI-Co and PANI-Pt hybrids (a) LM-Co and LM-Pt; (b) EM-Co and EM-Pt; (c) PNA-Co and PNA-Pt. The Co-based hybrids are on the top, while the Pt-based hybrids are on the bottom. (d) Comparison of the formation energies of Co- and Pt-based hybrids. 

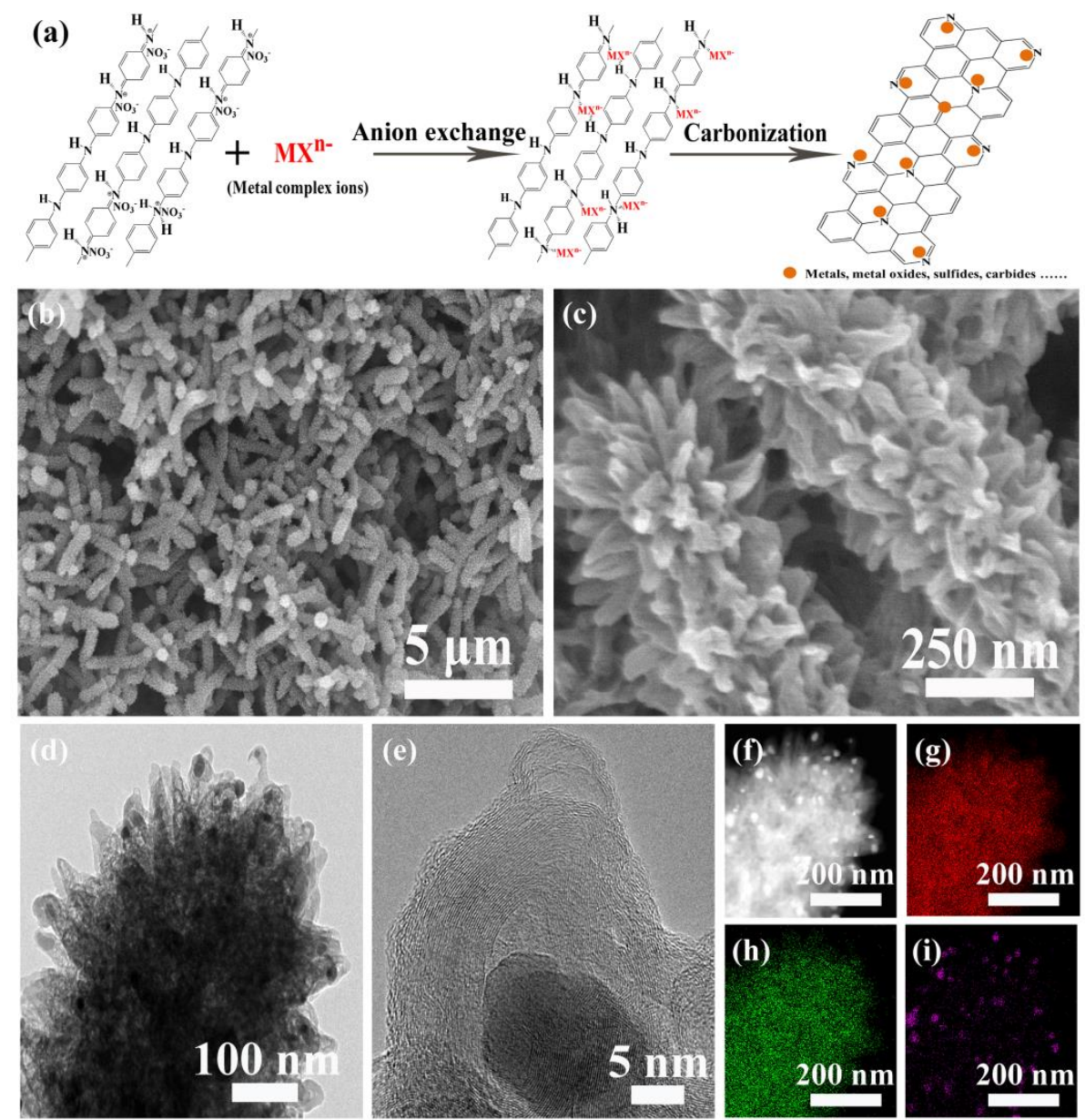

Fig. 4 (a) Schematic illustration of synthesis process of inorganic-carbon hybrids from metal complex ions and PANI. (b)-(e) SEM and TEM images of Co@NC(0.45). (f)-(i) EDX mapping of Co@NC(0.45): (f) STEM image, (g) C, (h) N, and (i) Co elements mapping. 

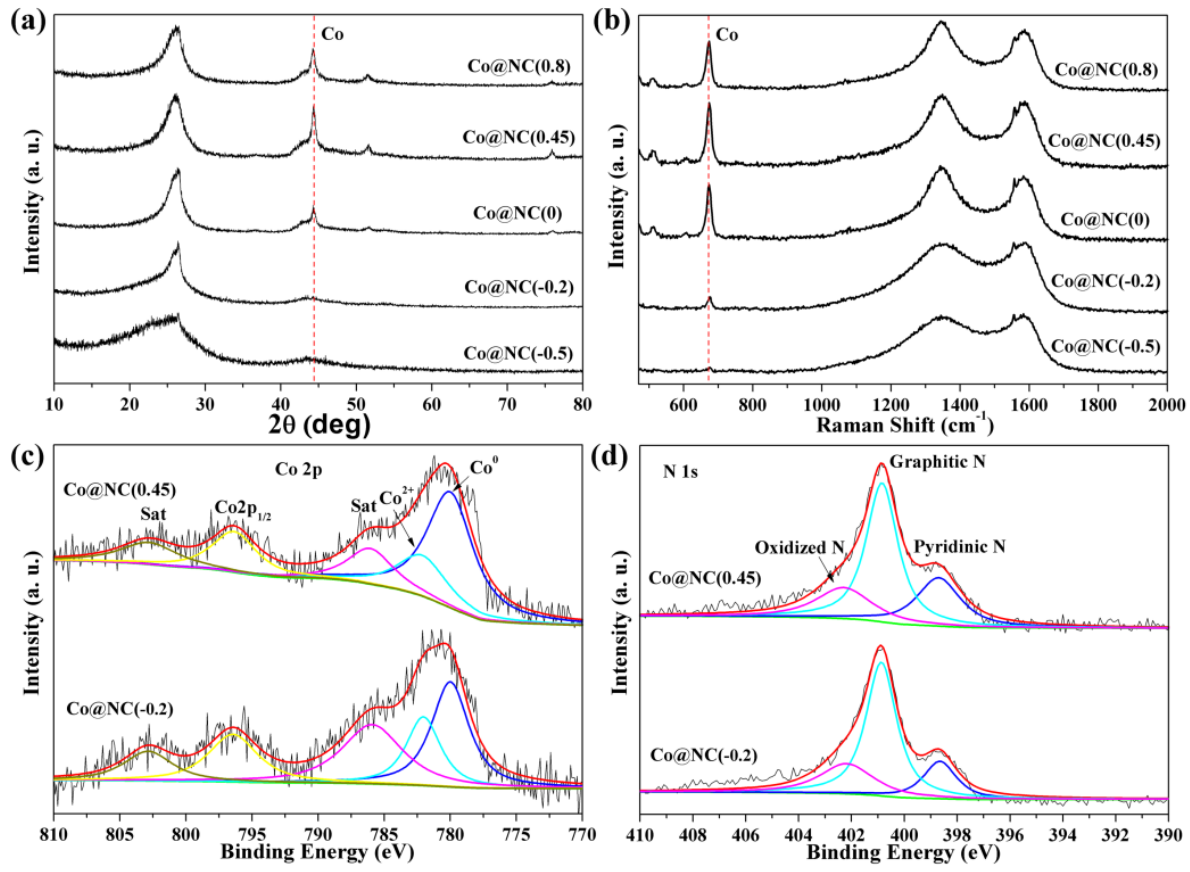

Fig. 5 (a) XRD patterns, (b) Raman spectra, (c) Co 2p and (d) N 1s XPS spectra of the Co@ NC samples. 

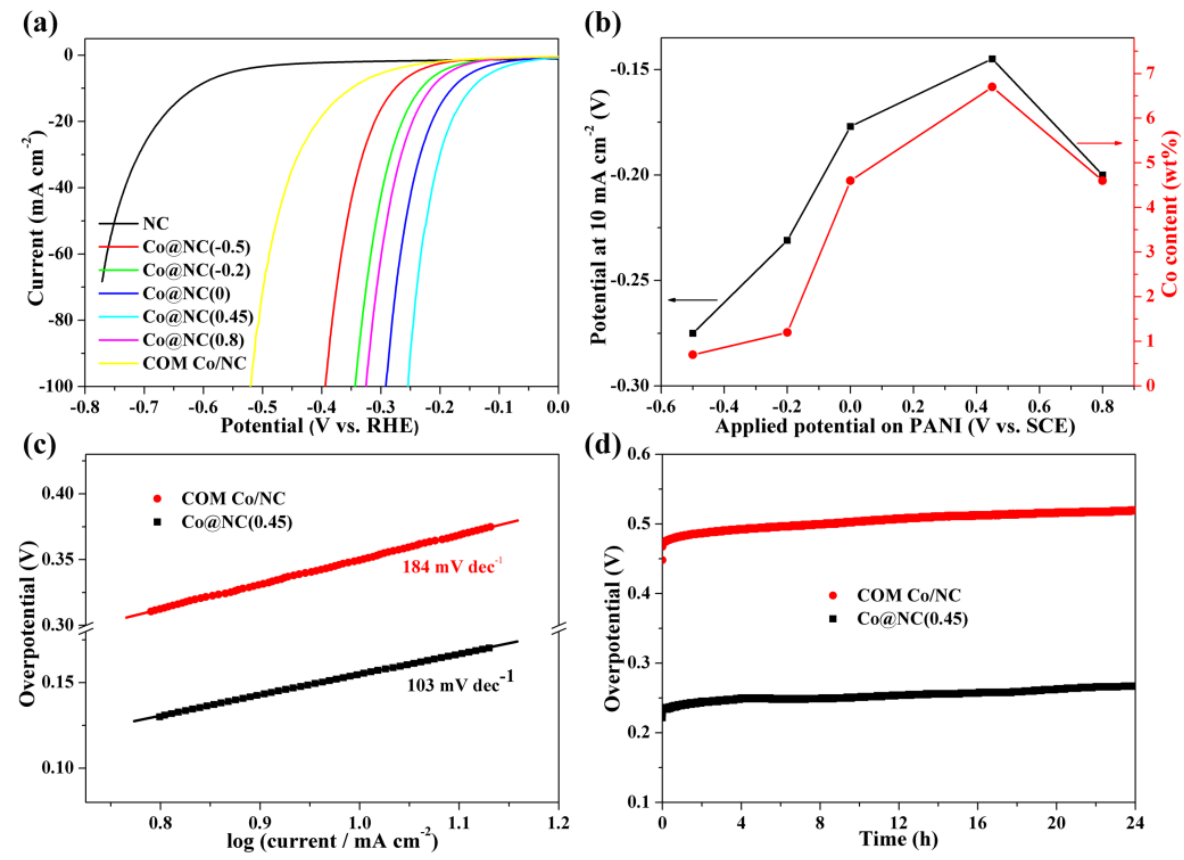

Fig. 6 (a) Polarization curves of a series of Co@NC samples prepared by applying different potentials on PANI from $-0.5 \mathrm{~V}$ to $0.8 \mathrm{~V}$ and commercial PANI derived $\mathrm{Co} / \mathrm{NC}$ sample $(\mathrm{COM} \mathrm{Co} / \mathrm{NC})$. (b) Potentials at $10 \mathrm{~mA} \mathrm{~cm}{ }^{-2}$ and metal $(\mathrm{Co})$ content as a function of the applied potentials. (c) Tafel plots of Co@ $\mathrm{NC}(0.45)$ and $\mathrm{COM} \mathrm{Co} / \mathrm{NC}$. (d) Chronopotentiometric curves of $\mathrm{Co} @ \mathrm{NC}(0.45)$ and $\mathrm{COM} \mathrm{Co} / \mathrm{NC}$ with a constant current densities of $50 \mathrm{~mA} \mathrm{~cm}^{-2}$. 

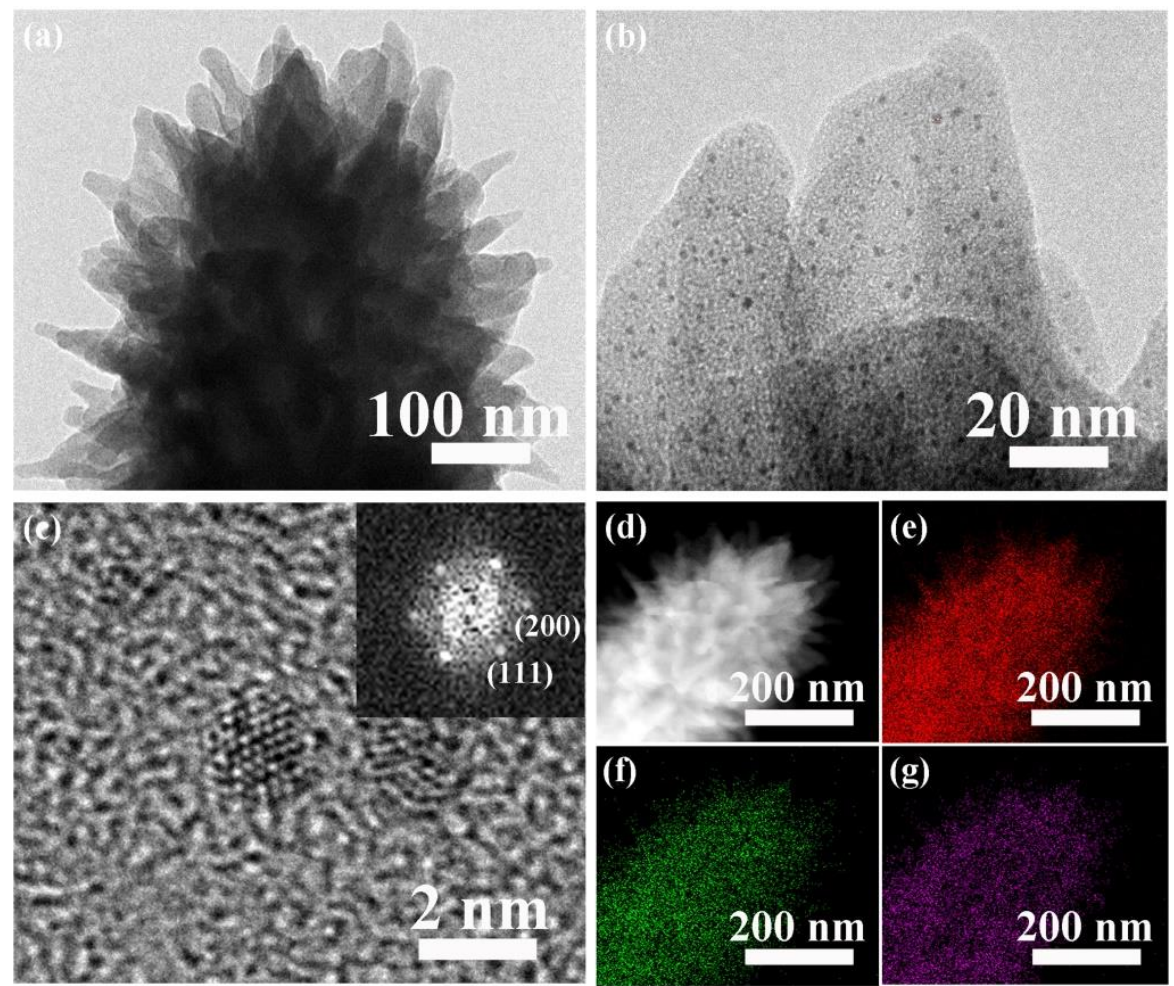

Fig. 7 (a) and (b) TEM images and (c) HRTEM image of Pt@NC(-0.2). Inset of (c) is the corresponding FFT pattern. (d)-(g) EDX mapping of Pt@NC(-0.2): (d) STEM image, (e) C, (f) N, and (g) Pt elements mapping. 

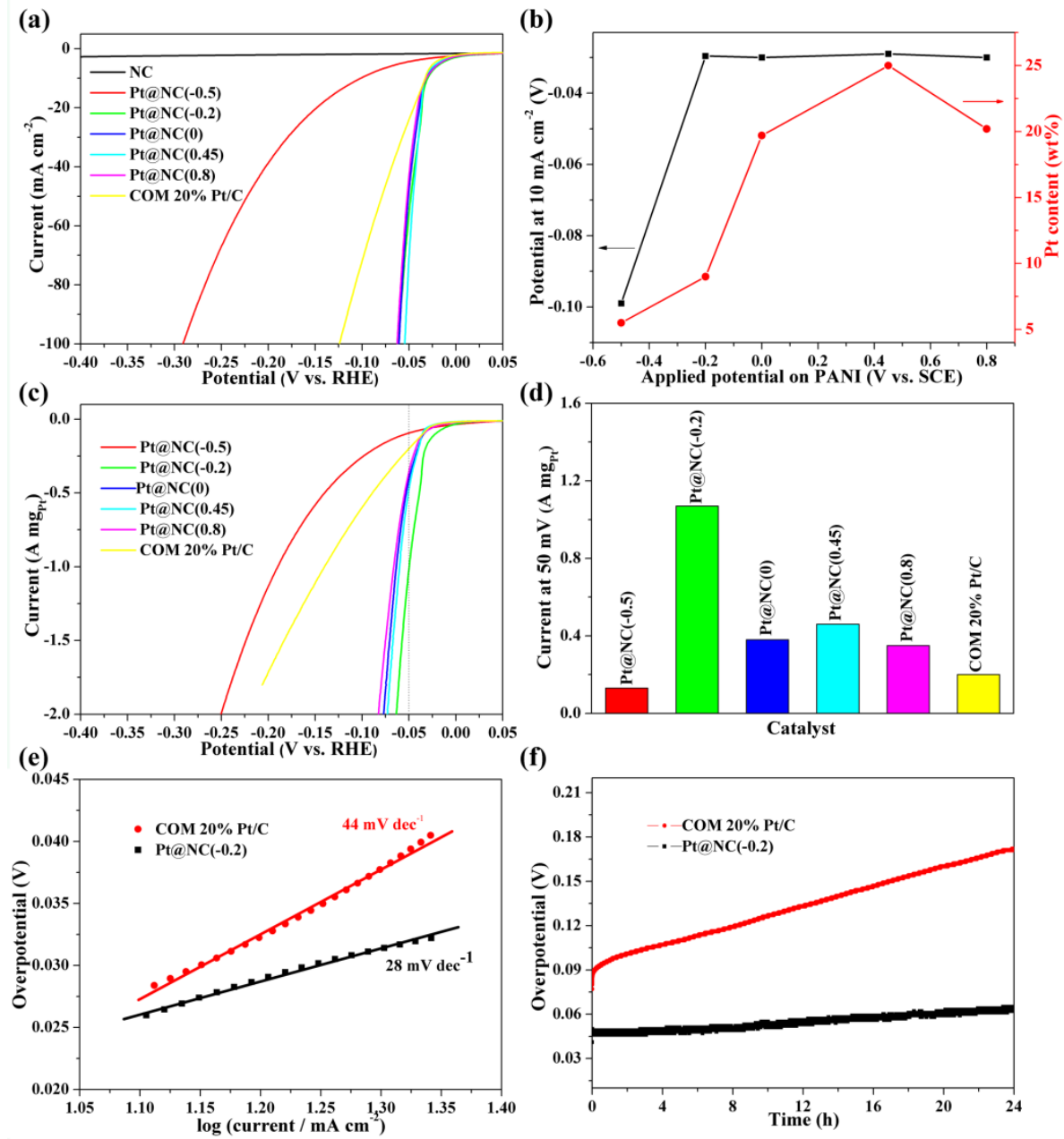

Fig. 8 (a) Polarization curves of a series of Pt@NC samples prepared by applying different potentials on PANI from $-0.5 \mathrm{~V}$ to $0.8 \mathrm{~V}$ and commercial $20 \mathrm{wt} \% \mathrm{Pt} / \mathrm{C}(\mathrm{COM} 20 \% \mathrm{Pt} / \mathrm{C})$. (b) Potentials at $10 \mathrm{~mA} \mathrm{~cm}{ }^{-2}$ and metal (Pt) content as a function of the applied potentials on PANI. (c) Polarization curves of Pt@NC samples and COM 20\% Pt/C based on the Pt metal mass. (d) Comparison of mass activities by the current densities of (c) at the overpotential of $50 \mathrm{mV}$. (e) Tafel plots of Pt@ NC(-0.2) and COM 20\% Pt/C. (f) Chronopotentiometric curves of Pt@NC(-0.2) and COM 20\% Pt/C with a constant current densities of 50 $\mathrm{mA} \mathrm{cm}{ }^{-2}$. 